\title{
Clonality in Sclerotinia sclerotiorum on Infected Cabbage in Eastern North Carolina
}

\author{
M. A. Cubeta, B. R. Cody, Y. Kohli, and L. M. Kohn
}

First and second authors: Department of Plant Pathology, North Carolina State University, Plymouth 27692; and third and fourth authors: Department of Botany, Erindale College, University of Toronto, Mississauga, Ontario, Canada L5L 1C6.

Accepted for publication 8 July 1997.

\begin{abstract}
Cubeta, M. A., Cody, B. R., Kohli, Y., and Kohn, L. M. 1997. Clonality in Sclerotinia sclerotiorum on infected cabbage in eastern North Carolina. Phytopathology 87:1000-1004.

Eighty-four isolates of Sclerotinia sclerotiorum from four cabbage production fields in North Carolina and 16 isolates from an experimental cabbage field plot in Louisiana were DNA-fingerprinted and tested for mycelial compatibility. In a comparison with 594 unique DNA fingerprints of S. sclerotiorum from Canadian canola, no fingerprints were shared among Canadian, North Carolina, and Louisiana populations. DNA fingerprints from the North Carolina sample were distinctive from those of the Canadian and Louisiana samples, with significantly more hybridizing fragments in the 7.7- to 18-kilobase range. Forty-one mycelial com-

were identified from the Louisiana sample. From the North Carolina sample, 32 MCGs were each associated with a unique fingerprint; of these, there were 11 clones (i.e., cases in which two or more isolates belonged to the same MCG and shared the same DNA fingerprint). Six clones sampled from two or more fields represented approximately $29 \%$ of the total sample ( 24 of 84 isolates), with six clones recovered from fields $75 \mathrm{~km}$ apart. There were 10 cases in which one MCG was associated with more than one DNA fingerprint and two cases in which one DNA fingerprint was associated with more than one MCG. The small sample from Louisiana was strictly clonal. The North Carolina sample had a clonal component, but deviated from one-to-one association of MCG with DNA fingerprint to an extent consistent with more recombination or transposition than the other two populations sampled.
\end{abstract} patibility groups (MCGs) and 50 unique DNA fingerprints were identified from the North Carolina sample. Three MCGs and three fingerprints

The cosmopolitan soilborne fungus Sclerotinia sclerotiorum (Lib.) de Bary is an important pathogen on more than 408 different plant species (4). Although there is extensive literature on the biology and ecology of $S$. sclerotiorum $(1,18,20)$, information about the population biology of $S$. sclerotiorum is still emerging.

Recent studies by Kohn and coworkers $(8,10,11,14)$ have shown that, in Canada, each field crop of canola (Brassica napus L. or B. rapa L.) is infected by many clones of $S$. sclerotiorum. In these studies, a clone was defined as a genotype maintained in a single mitotic lineage $(2,11)$. Members of a clone shared four presumably independent molecular characteristics (5) and were identified by two putatively independent criteria, mycelial compatibility and DNA fingerprinting. All members of a clone were mycelially compatible and shared a unique DNA fingerprint. The DNA fingerprinting element, pLK44.20, used by Kohn and coworkers is repeated and dispersed in the nuclear genome (14) with high amino acid similarity to a non-long terminal repeat retrotransposon (8). That this putative transposable element is relatively stable is supported in two ways. First, isolates representing clonal genotypes that have been stored and serially cultured from 1989 to the present have maintained stable fingerprints with one notable exception, the loss of one hybridizing fragment in the fingerprint of DNA from a sector of one isolate (Y. Kohli, unpublished data). Second, the same clonal genotypes, identified both by fingerprinting and mycelial compatibility, have been recovered repeatedly over geographically long distances and over several years (11).

For example, in a one-season sample, 39 clones were identified among 66 isolates of $S$. sclerotiorum from seven locations in Alberta, Saskatchewan, and Manitoba. The most widely distributed

Corresponding author: M. A. Cubeta; E-mail address: cub@plymouth.ces.ncsu.edu

Publication no. P-1997-0815-01R

(C) 1997 The American Phytopathological Society
Additional keywords: crucifer, head rot, population biology, white mold. clone accounted for $18 \%$ of the isolates (10). Over the entire 4year sampling period, 659 clones were identified among the 2,876 $S$. sclerotiorum isolates sampled, with one clone recovered frequently and in every year of sampling over a distance of more than $2,000 \mathrm{~km}$. A small number of clones composed the majority of the sample, indicating that there is one large $S$. sclerotiorum population on Canadian canola. There were, however, a large number of genotypes sampled only once or twice (11). The strongly clonal structure of this population is consistent with efficient asexual reproduction via sclerotia and selfed sexual reproduction, with new clones arising through mutation and, perhaps, infrequent outbreeding.

Is this clonal population structure typical of S. sclerotiorum on other crops? Are the same Canadian canola clones found on other related crops such as cabbage? Head rot of cabbage (Brassica oleracea L. var. capitata), caused by $S$. sclerotiorum, is a common disease that occurs in most cabbage production regions of the world during periods of cool, wet weather. In eastern North Carolina, head rot of cabbage occurs primarily in the fall production season and can result in serious economic losses in the field and during transport to the market.

There were two main objectives in this study. First, to determine whether, like the Canadian canola population, field populations of S. sclerotiorum on cabbage in eastern North Carolina are predominantly clonal, and whether each cabbage field is infected by many clones. This entailed determining whether the relatively strict association between mycelial compatibility grouping and DNA fingerprint observed in the Canadian canola field populations also occurred in North Carolina cabbage field populations. Strict association between these two criteria would indicate clonality mediated by asexual reproduction and selfed sexual reproduction, while a lack of association would be consistent with transposition, or genetic exchange and recombination. The second objective was to determine whether the same genotypes of S. sclerotiorum were found associated with Canadian canola and North 
Carolina cabbage. A small sample from one Louisiana cabbage field population was also included in the study.

\section{MATERIALS AND METHODS}

Isolates. Eighty-four S. sclerotiorum isolates were obtained from naturally infected cabbage from four production fields in eastern North Carolina (Table 1). Individual cabbage heads exhibiting head rot symptoms, at least $1 \mathrm{~m}$ apart, were arbitrarily collected during the second week of November 1994. Ten different cabbage heads from fields 1,2 , and 4 (30 total), and 54 different cabbage heads from field 3 (the most intensively sampled field) were used for isolations. One isolate was obtained from each head by surface-disinfesting a single sclerotium for $1 \mathrm{~min}$ in $70 \%$ ethyl alcohol, rinsing in sterile distilled water, and then plating on potato dextrose agar (PDA) (Difco Laboratories, Detroit) amended with streptomycin sulfate and tetracycline $(50 \mu \mathrm{g} / \mathrm{ml}$ of each) (Sigma Chemical Co., St. Louis). Isolates were maintained by storing sclerotia in glass vials in the dark at $-20^{\circ} \mathrm{C}$.

For a comparative southern U.S. cabbage sample, a small sample made by G. Holcomb from a 0.2-ha experimental cabbage field plot at the Burden Research Plantation, Louisiana State University, Baton Rouge, in 1996, was also evaluated. Four cabbage heads with head rot symptoms were sampled approximately 17 to $33 \mathrm{~m}$ apart. The cabbage heads were designated Lou1, Lou2, Lou3, and Lou4, such that a sclerotium collected from cabbage head 1 was designated Lou1-1. Five isolates were obtained from each cabbage head. With only one viable isolate recovered for Lou1, the total number of Louisiana cabbage isolates screened was 16.

Mycelial compatibility grouping. To determine the association between mycelial compatibility group (MCG) and DNA fingerprint, whether fields supported more than one genotype, and whether some genotypes were found in more than one field, mycelial compatibility was assessed in three subsamples by pairing isolates in all possible combinations using the procedure of Kohn et al. (12). All pairings were scored after incubation in the dark at $23^{\circ} \mathrm{C}$ for 4,7 , and 14 days. Pairings were scored as mycelially compatible when no reaction line was observed in the interaction zone between paired isolates. Pairings were scored as mycelially incompatible when a reaction line of either hyphal tufts or red pigment deposition was observed between paired isolates (12). Pairings were scored independently by at least two people. Testing in each subsample was repeated at least twice.

DNA fingerprinting. DNA fingerprinting was conducted as described previously (8). Whole cell genomic DNAs of all isolates were digested with $B a m \mathrm{HI}$ and then hybridized to a clone probe, pLK44.20 (14). DNAs from North Carolina and Louisiana cabbage isolates were subjected to electrophoresis at $1.5 \mathrm{~V} / \mathrm{cm}$ for 45 to $48 \mathrm{~h}$, rather than 16 to $24 \mathrm{~h}$ for Canadian canola isolates, to resolve the characteristically clustered hybridizing fragments. Fingerprints of North Carolina cabbage and Canadian canola isolates were scored with reference to a Canadian canola isolate, LMK 211 , used as a size standard on all gels in this study and previous studies in the Kohn laboratory $(8,13,14)$. Hybridizing fragments were binned in 53 size classes (8) and binomially coded as present or absent.

In this study, each genotype was characterized by a unique DNA fingerprint. A clone was defined as a group of isolates ( $>1$ isolate) that shared a unique DNA fingerprint and belonged to the same MCG (i.e., isolates were all mycelially compatible with each other, but incompatible with isolates from other MCGs).

\section{RESULTS}

Mycelial compatibility grouping and DNA fingerprinting. Forty-one MCGs were designated from the North Carolina sample and three MCGs from the Louisiana sample (Table 1). Ninetyeight percent of the North Carolina pairings were scored identically
TABLE 1. DNA fingerprint designations and frequencies associated with mycelial compatibility group (MCG) of Sclerotinia sclerotiorum isolates from infected cabbage heads sampled from each of four fields in North Carolina and one field in Louisiana

\begin{tabular}{|c|c|c|c|c|c|}
\hline \multirow[b]{3}{*}{$\mathrm{MCG}^{\mathrm{d}}$} & \multicolumn{5}{|c|}{ DNA fingerprint designation and frequency ${ }^{a}$} \\
\hline & \multicolumn{4}{|c|}{ North Carolina ${ }^{b}$} & \multirow{2}{*}{$\frac{\text { Louisiana }^{\mathrm{c}}}{\text { Field } 5}$} \\
\hline & Field 1 & Field 2 & Field 3 & Field 4 & \\
\hline $01 * \mathrm{e}$ & & & $17(4), 18(1)$ & & \\
\hline $02 *$ & & & $20(1), 35(4)$ & & \\
\hline $03 *$ & $39(1)$ & $37(1)$ & $37(3)$ & $37(2)$ & \\
\hline $04 *$ & & & 03 (1), 08 (1) & & \\
\hline $05^{*}$ & & & $09(1), 12(1)$ & & \\
\hline 06 & $27(1)$ & $27(2)$ & $27(1)$ & & \\
\hline $07 *$ & $47(2)$ & & $47(1), 48(1)$ & & \\
\hline 08 & $01(1)$ & & & $01(1)$ & \\
\hline 09 & & $23(2)$ & & & \\
\hline 10 & & $15(1)$ & $15(2)$ & $15(2)$ & \\
\hline 11 & & & $43(2)$ & $43(2)$ & \\
\hline $12 *$ & $29(1)$ & & $51(1)$ & & \\
\hline $13 *$ & & & $26(1), 52(1)$ & & \\
\hline 14 & & & $01(2)$ & & \\
\hline 16 & & & $30(3)$ & & \\
\hline 17 & & & $14(1)$ & & \\
\hline 18 & $01(1)$ & & & & \\
\hline 19 & $32(1)$ & & & & \\
\hline
\end{tabular}

20

21

23

24

27

28

29

$30^{*}$

32

33

34

37

39
40

41

44

45

46

47
48

49

50

51

52

$\begin{array}{ll} & 25(1) \\ & 45(1) \\ 06(1) & 07(1) \\ 34(1) & \\ 44(1) & \\ 05(1) & \\ 17(1) & \\ 53(1) & \\ 38(1) & \\ 33(1) & \\ 21(1) & \\ 19(1) & \\ 11(1) & \\ 31(1) & \\ 36(1) & \\ 42(1) & \\ 02(1) & \\ 46(1) & \end{array}$

22 (1)

54 (4)

$55(7)$

56 (5)

$n^{\mathrm{f}} \quad 10$

10

10

54

10

16

a DNA fingerprint was determined from Southern hybridizations of restrictiondigested genomic DNA with pLK44.20, which contains a homologous, dispersed repetitive element. Isolates with the same fingerprint designation number have an identical DNA fingerprint. Fingerprint frequency is the number of isolates sampled with a unique DNA fingerprint that belonged to the designated MCG. Frequencies are in parentheses, e.g., for MCG 01 in Field 3, four isolates had fingerprint 17 and one isolate had fingerprint 18

${ }^{\mathrm{b}}$ Commercial cabbage production fields in North Carolina, 1994. Fields 1 (cv. Bravo) and 2 (cv. Market Prize) were $4 \mathrm{~km}$ from each other in Plymouth, and fields 3 (cv. Quisto) and 4 (cv. Bravo) were $3 \mathrm{~km}$ from each other in Weeksville. Plymouth is approximately $75 \mathrm{~km}$ from Weeksville. Each isolate was made from one sclerotium removed from one infected cabbage head. After surface-disinfestation, sclerotia were placed on potato dextrose agar amended with streptomycin sulfate and tetracycline $(50 \mu \mathrm{g} / \mathrm{ml}$ of each).

${ }^{c}$ Field 5 (cv. unknown) was an experimental cabbage field plot, Burden Research Plantation, Louisiana State University, Baton Rouge, 1996. Five isolates were made from each of five sclerotia; five sclerotia were sampled for each of four cabbage heads.

d MCG determined by pairing isolates on modified Patterson's medium (12). Isolates with the same MCG number were mycelially compatible.

e Asterisks indicate each of nine MCGs associated with more than one DNA fingerprint. There were two examples of one fingerprint associated with more than one MCG: MCGs 01 and 30 were both associated with fingerprint 17, and MCGs 08, 14, and 18 were all associated with fingerprint 01 .

${ }^{f}$ Sample size. 
by all evaluators; $2 \%$ of the pairings were difficult to score and were repeated in both the Cubeta and Kohn laboratories, scored by four people, and paired again if necessary until agreement was reached on the scoring. Louisiana pairings were unambiguous.

Fifty DNA fingerprints were identified among the North Carolina isolates and three fingerprints from the Louisiana sample. Sample fingerprints are shown in Figure 1. There were no matches of DNA fingerprints in comparisons of fingerprints of Canadian canola isolates with North Carolina and Louisiana cabbage isolates. From the North Carolina sample, 32 MCGs were associated with one fingerprint; of these, there were 11 clones (i.e., cases in which two or more isolates belonged to the same MCG and shared the same DNA fingerprint). Six clones sampled from two or more fields represented approximately $29 \%$ of the total sample (24 of 84 isolates). There were 10 cases in which one MCG was associated with more than one fingerprint (Table 2 ) and two cases in which one fingerprint was associated with more than one MCG (additional DNA fingerprint data available upon request).

Direct comparison of individual hybridizing fragments in fingerprints between the samples was complicated by the greater number of fragments in the North Carolina sample, between 7.7 and
18 kilobases $(\mathrm{kb})$. Resolution of these hybridizing fragments required different electrophoretic conditions from those previously employed for the large Canadian canola studies, although the Canadian standard isolate used in scoring, LMK 211, was included on all gels. To make comparisons of size classes of fragments, a subsample of 84 isolates from the total Canadian canola sample was selected using random numbers. The proportion of fragments between 7.7 and $18 \mathrm{~kb}$ was $57.9 \%$ for the North Carolina sample and $35.1 \%$ in the Canadian canola subsample (Table 3). There were no fragments $>25 \mathrm{~kb}$ in the North Carolina sample. One fragment in this size class represented a proportion of $8.0 \%$ of hybridizing fragments in the Canadian canola subsample. The chi-square statistic for frequency of occurrence of fragments in three size classes ( 0 to $5 \mathrm{~kb}, 5$ to $13 \mathrm{~kb}$, and >13 kb) between North Carolina and Canada was highly significant $\left(x^{2}=\right.$ 30.618 , df $=2, P<0.001$ ). Interpolation of fragments between 7.7 and $18 \mathrm{~kb}$ against the Canadian standard was ruled out for two reasons. First, interpolating against the standard was difficult in this congested size class of hybridizing fragments. Second, we did not automatically accept the basic assumption that comigrating fragments, finely distinguished from a congested group of other fragments, share common descent in this interpopulational comparison.

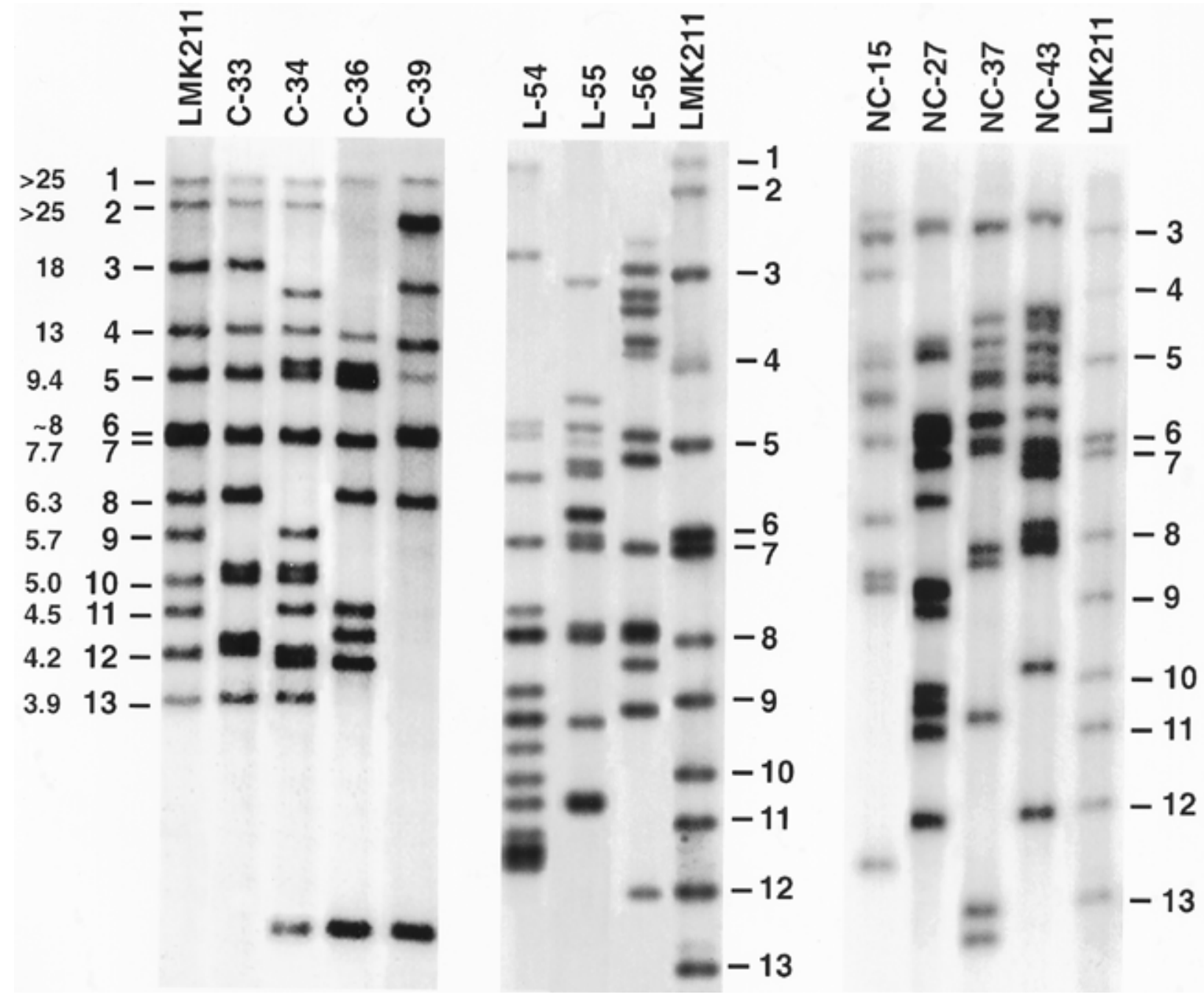

Fig. 1. DNA fingerprints of clones of Sclerotinia sclerotiorum from Canadian canola (C-33, C-34, C36, and C-39), Louisiana cabbage (L-54, L-55, and L56), and North Carolina cabbage (NC-15, NC-27, NC-37, and NC-43). A clone is defined as a group of isolates (>1 isolate) that share a unique DNA fingerprint and are mycelially compatible with each other, but incompatible with isolates of other clones. Fingerprints were obtained by Southern hybridization of BamHI-digested genomic DNA with pLK44.20, a plasmid carrying a dispersed repetitive element. DNA of reference isolate LMK 211, from Canadian canola, was used as a reference for all DNA fingerprints in this study. Numbers 1 to 13 designate each hybridizing fragment of LMK 211; the sizes of these fragments are indicated in kilobases on the left. Fingerprints were scanned from one autoradiogram each from Canadian canola, Louisiana cabbage, and North Carolina cabbage, respectively. 


\section{DISCUSSION}

In comparison to the extensive samples of S. sclerotiorum from Canadian canola that were strongly clonal, i.e., more than three clones represented $>50 \%$ of the total sample $(8,10,14)$, the North Carolina sample had a clonal component (six clones represented $29 \%$ of the total sample of 84 isolates) and the small Louisiana sample was strictly clonal (three clones represented $100 \%$ of 16 isolates). As in the Canadian sample, each field in North Carolina and the Louisiana field was infested with many genotypes of S. sclerotiorum. In both Canadian and North Carolina samples, a few genotypes were recovered at a higher frequency, and many genotypes were sampled only once. As in Canada, genotypes in North Carolina may be dispersed across geographically long distances (over $75 \mathrm{~km}$ ) in North Carolina.

The deviations from strict association between MCG and DNA fingerprint in the North Carolina sample were notable. In a Canadian sample of 66 isolates from seven locations (10), 32 MCGs each were associated with one unique fingerprint, while three MCGs included isolates with one of two different fingerprints. In the North Carolina sample, there were 10 such cases, with MCG 04 associated with four different fingerprints. In no case among the $\mathrm{Ca}-$ nadian sample was one fingerprint associated with more than one MCG, as was observed in the North Carolina sample with fingerprints 01 and 17. Among the Canadian isolates, no cases were observed of nontransitivity in mycelial compatibility testing, e.g., isolate $\mathrm{A}$ is compatible with isolate $\mathrm{B}$, isolate $\mathrm{B}$ is compatible with isolate $\mathrm{C}$, but isolate $\mathrm{A}$ is not compatible with isolate $\mathrm{C}$. One such case was observed with MCGs 01 and 30 in the North Carolina sample.

Also notable in the North Carolina sample were the ambiguous compatibility reactions among $2 \%$ of the mycelial pairings. In mycelial compatibility tests of Canadian and Louisiana isolates, incompatibility was conclusively demonstrated by a red reaction line

TABLE 2. DNA fingerprints associated with the 10 mycelial compatibility groups (MCGs) of Sclerotinia sclerotiorum isolates from cabbage in eastern North Carolina for which more than one unique fingerprint is associated with a single MCG

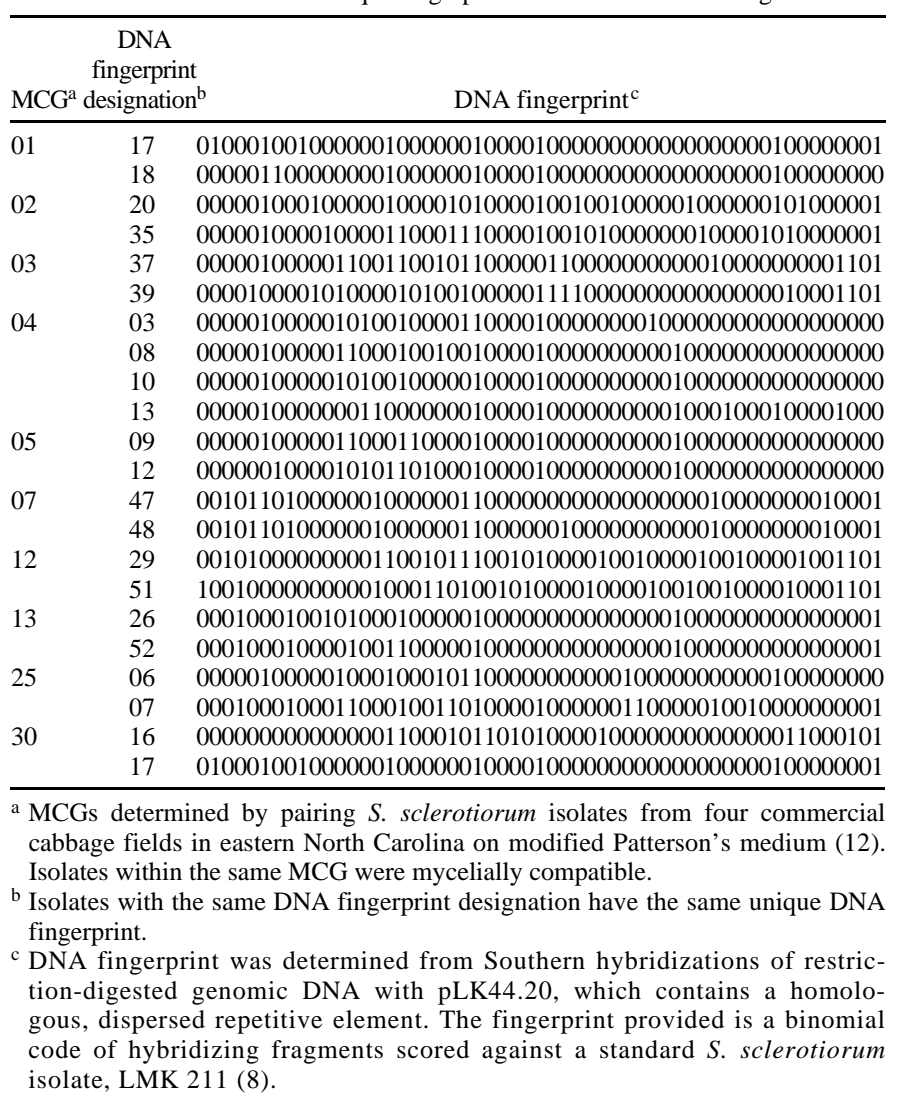

in the interaction zone. Isolates with the same DNA fingerprint were unambiguously compatible. Among some groups of isolates with the same fingerprint in the North Carolina sample, however, we observed a range of compatibility reactions from full compatibility to the formation of vague reaction lines of thin or fluffy hyphae. Mycelial compatibility, the ability of two mycelia to anastomose and form one mycelium, is probably a component of vegetative compatibility, in which two mycelia anastomose to form a stable heterokaryon. Vegetative compatibility in the few ascomycetes that have been investigated is determined by several loci. For two isolates to be vegetatively compatible, all vegetative compatibility loci, which are allelic in some ascomycetes, must be the same $(7,15)$. The genetic regulation of mycelial compatibility in $S$. sclerotiorum is not known, but our observations are consistent with partial compatibility mediated by the sharing of some, but not all, compatibility loci or alleles. This could be best explained by the shuffling of mycelial compatibility loci or alleles in recombination. It would be difficult to distinguish genetic exchange in somatic heterokaryons from outbreeding in sexual reproduction. Pairings of isolates derived from sibling ascospores from each of 12 apothecia from field populations of North Carolina cabbage provided some evidence of mycelial incompatibility, consistent with recombination, from two apothecia. However, DNA fingerprints of the sibling ascospores were all identical (M. A. Cubeta and L. M. Kohn, unpublished data).

While the range of mycelial compatibility reactions suggest recombination, the deviations from one-to-one association of MCG to fingerprint are consistent with recombination or transposition. Because pLK44.20 contains a repeated, dispersed DNA element, transposition must be considered. It is possible that rates of transposition vary among subpopulations of $S$. sclerotiorum. The data presented in Table 2 allow comparison of DNA fingerprints associated with a single MCG. DNA fingerprints for isolates belonging to one MCG may differ by as few as one or two hybridizing fragments or by five or more fragments (e.g., DNA fingerprints associated with MCG 04), suggesting a greater role for transposition within MCGs of the North Carolina sample. To rigorously address this, and to compare the contributions of recombination and mutation, we are using phylogenetic analyses of single nucleotide substitutions in several genomic DNA regions for each population sample (I. Carbone and L. M. Kohn, unpublished data). DNA sequence-based screens of population samples provide characters that are amenable to phylogenetic reconstruction and are less

TABLE 3. Distribution of hybridizing fragments based on size class ${ }^{\text {a }}$ from DNA fingerprints of isolates of Sclerotinia sclerotiorum from the North Carolina cabbage sample and a random subsample of the same size from the Canadian canola population

Fragment size range $(\mathrm{kb})$ Number of fragments ${ }^{\mathrm{b}}$ Proportion of fragments ${ }^{\mathrm{b}}$

\begin{tabular}{lcc}
\hline$<2.2$ & $2(0)$ & $0.004(0.000)$ \\
$2.2-3.9$ & $2(4)$ & $0.039(0.120)$ \\
$4-4.2$ & $5(4)$ & $0.054(0.062)$ \\
$4.3-4.5$ & $4(4)$ & $0.029(0.051)$ \\
$4.6-5$ & $3(3)$ & $0.037(0.031)$ \\
$5.1-5.7$ & $7(2)$ & $0.058(0.034)$ \\
$5.8-6.3$ & $5(4)$ & $0.125(0.103)$ \\
$6.4-7.7$ & $4(3)$ & $0.134(0.108)$ \\
$7.8-9.4$ & $7(6)$ & $0.212(0.163)$ \\
$9.5-13$ & $7(4)$ & $0.156(0.085)$ \\
$13.1-18$ & $4(6)$ & $0.111(0.108)$ \\
$18.1-25$ & $4(4)$ & $0.039(0.051)$ \\
$>25$ & $0(1)$ & $0.000(0.082)$ \\
& $54(45)$ & $0.998(0.998)$
\end{tabular}

a Hybridizing fragments were assigned to size classes by comparison to a reference isolate, LMK 211, from Canadian canola (10), which was included on every gel in this study.

b Data from the North Carolina cabbage sample $(n=84)$. Numbers in parentheses are data from a random subsample $(n=84)$ of the Canadian canola population of 2,747 isolates $(8,11)$. 
burdened by the assumptions that must be made with comigrating bands in electrophoresis.

Six clones, composing 24 isolates or $29 \%$ of the sample, were recovered from more than one field in the North Carolina sample. The distribution of some genotypes may result from movement of sclerotia in seed and in soil on equipment and with cabbage transplants $(17,19)$. The movement and immigration of infected seed has been implicated in the geographic distribution and occurrence of Mycosphaerella graminicola (16) and Phytophthora infestans (6). Ben-Yephet and Bitton (3) and Williams and Stelfox (21) reported that ascospores of $S$. sclerotiorum can be dispersed several hundred meters, but these studies did not utilize labeled propagules that could be rigorously traced. Ben-Yephet and Bitton (3) estimated that approximately 77 to $90 \%$ of the ascospores were deposited within $100 \mathrm{~m}$ from the apothecial source, while the remaining ascospores were transported farther. While fungal propagules associated with aerial dispersal are often melanized and thickwalled, ascospores of $S$. sclerotiorum are hyaline and relatively thin-walled. We think that, while the airborne movement of ascospores for distances of 3 to $4 \mathrm{~km}$ is possible, the $75 \mathrm{~km}$ distance between Plymouth and Weeksville is unlikely.

Based on DNA fingerprints, there were no genotypes of S. sclerotiorum shared among the Canadian canola, North Carolina, and Louisiana population samples. In this study, each fingerprint was compared as a phenotype. Because of the difficulty in inferring common descent from common state, we have avoided comparisons of comigrating hybridizing fragments, especially between population samples. These data are consistent with a population of $S$. sclerotiorum subdivided by geographic separation, host specialization, or ecological adaptation. The absence of commercial cabbage and canola seed production in North Carolina may have contributed to the isolation of local genotypes of $S$. sclerotiorum. Population subdivision could be important in customizing disease management, especially if it is associated with local ecological adaptation. However, all that can be conclusively interpreted from these data is that there are no genotypes of $S$. sclerotiorum shared among these locations. In subsamples from the same populations in our study, three mitochondrial haplotypes, determined by hybridization of mitochondrial DNA (mtDNA) with whole genomic DNA, were shared by $S$. sclerotiorum isolates from Canadian canola and North Carolina cabbage (9). One mtDNA haplotype was shared among all agricultural populations sampled worldwide (Australia and Norway, as well as Canada and North Carolina), but absent from two wild populations. The hypothesis that agricultural populations of $S$. sclerotiorum share a relatively recent, common mitochondrial origin and that the worldwide population is subdivided awaits further study.

\section{ACKNOWLEDGMENTS}

We thank R. G. Upchurch and M. L. Carson for presubmission reviews; P. E. Puryear for assistance in literature searches; and W. G. Meads, P. Lowry, and L. Small for providing assistance in locating cabbage fields.

\section{LITERATURE CITED}

1. Adams, P. B., and Ayers, W. A. 1979. Ecology of Sclerotinia species. Phytopathology 69:896-899.

2. Anderson, J. B., and Kohn, L. M. 1995. Clonality in soilborne, plantpathogenic fungi. Annu. Rev. Phytopathol. 33:369-391.

3. Ben-Yephet, Y., and Bitton, S. 1985. Use of a selective medium to study the dispersal of ascospores of Sclerotinia sclerotiorum. Phytoparasitica 13:33-40.

4. Boland, G. J., and Hall, R. 1994. Index of plant hosts for Sclerotinia sclerotiorum. Can. J. Bot. 16:93-108.

5. Errampalli, D., and Kohn, L. M. 1995. Comparison of pectic zymograms produced by different clones of Sclerotinia sclerotiorum in culture. Phytopathology 85:292-298.

6. Fry, W. E., Goodwin, S. B., Matuszak, J. M., Spielman, L. J., and Milgroom, M. G. 1992. Population genetics and intercontinental migrations of Phytophthora infestans. Annu. Rev. Phytopathol. 30:107-129.

7. Glass, N. L., and Kuldau, G. A. 1992. Mating type and vegetative incompatibility in filamentous Ascomycetes. Annu. Rev. Phytopathol. 30: 201-224.

8. Kohli, Y., Brunner, L. J., Yoell, H., and Kohn, L. M. 1995. Clonal dispersal and spatial mixing in populations of the plant pathogenic fungus, Sclerotinia sclerotiorum. Mol. Ecol. 4:69-77.

9. Kohli, Y., and Kohn, L. M. 1996. Mitochondrial haplotypes in populations of the plant-infecting fungus Sclerotinia sclerotiorum: Wide distribution in agriculture, local distributions in the wild. Mol. Ecol. 5:773-783.

10. Kohli, Y., Morrall, R. A. A., Anderson, J. B., and Kohn, L. M. 1992. Local and trans-Canadian clonal distribution of Sclerotinia sclerotiorum on canola. Phytopathology 82:875-880.

11. Kohn, L. M. 1995. The clonal dynamic in wild and agricultural plant pathogen populations. Can. J. Bot. 73(Suppl. 1):1231-1240.

12. Kohn, L. M., Carbone, I., and Anderson, J. B. 1990. Mycelial interactions in Sclerotinia sclerotiorum. Exp. Mycol. 14:255-267.

13. Kohn, L. M., Petsche, D. M., Bailey, S. R., Novak, L. A., and Anderson, J. B. 1988. Restriction fragment length polymorphisms in nuclear and mitochondrial DNA of Sclerotinia species. Phytopathology 78:10471051.

14. Kohn, L. M., Stasovski, E., Carbone, I., Royer, J., and Anderson, J. B. 1991. Mycelial incompatibility and molecular markers identify genetic variability in field populations of Sclerotinia sclerotiorum. Phytopathology 81:480-485.

15. Leslie, J. F. 1993. Fungal vegetative compatibility. Annu. Rev. Phytopathol. 31:127-150.

16. McDonald, B. A., and Martinez, J. P. 1990. DNA restriction fragment length polymorphisms among Mycosphaerella graminicola (anamorph Septoria tritici) isolates collected from a single wheat field. Phytopathology 80:1368-1373.

17. McLean, D. M. 1958. Role of dead flower parts in infection of certain crucifers by Sclerotinia sclerotiorum (Lib.) D. By. Plant Dis. Rep. 42:663-666.

18. Mitchell, S. J., and Wheeler, B. E. J. 1990. Factors affecting the production of apothecia and longevity of sclerotia of Sclerotinia sclerotiorum. Plant Pathol. 39:70-74.

19. Neergaard, P. 1958. Mycelial seed infection of certain crucifers by Sclerotinia sclerotiorum (Lib.) D. By. Plant Dis. Rep. 42:1105-1106.

20. Willets, H. J., and Wong, J. A. L. 1980. The biology of Sclerotinia sclerotiorum, S. trifoliorum and S. minor with emphasis on specific nomenclature. Bot. Rev. 46:101-165.

21. Williams, J. R., and Stelfox, D. 1979. Dispersal of ascospores of Sclerotinia sclerotiorum in relation to sclerotinia stem rot of rapeseed. Plant Dis. Rep. 63:395-399. 